\title{
Counting Tm Dopant Atoms in and Around GaN Dots Using Scannning Transmission Electron Microscopy
}

\author{
J.L. Rouvière*, H. Okuno**, P.H. Jouneau*, P. Bayle-Guillemaud* and B. Daudin*** \\ * CEA INAC, SP2M, LEMMA Minatec, 17 rue des Martyrs Grenoble, F-38054 France. \\ ** now at CEA, LITEN, DTNM, LCRE, 17 rue des Martyrs Grenoble, F-38054 France. \\ *** CEA INAC, SP2M, NPSC, 17 rue des Martyrs Grenoble, F-38054 France.
}

In order to improve the efficiency of luminescence of $\mathrm{GaN}$ based devices, it has been proposed to use rare-earth doped $\mathrm{GaN}$ quantum dots (QD) in order to confine rare-earth ions and electron-hole pairs in the QDs, far from defects [1]. In this case, the accurate incorporation and localization of dopants in the dots is crucial.

In this work, high resolution Z-contrast STEM imaging is used to study the Tm doping of GaN quantum dots grown in AlN by molecular beam epitaxy (MBE). HAADF STEM allows us to visualize directly individual $\mathrm{Tm}$ atoms in the AlN matrix and even to count the number of Tm atoms in a given AlN atomic column (Fig. 1). Determining the Tm content in a GaN dot is more difficult because, (i) one has to check that the dot is included in the TEM lamella and (ii) the difference in atomic number $\mathrm{Z}$, between $\mathrm{Tm}\left(\mathrm{Z}_{\mathrm{Tm}}=69\right)$ and $\mathrm{Ga}\left(\mathrm{Z}_{\mathrm{Ga}}=31\right)$ is smaller than between $\mathrm{Tm}$ and $\mathrm{Al}$ $\left(Z_{\mathrm{Al}}=13\right)$. We will discuss the possibility to obtain $3 \mathrm{D}$-information from focal series.

We introduce a new visibility coefficient to determine quantitatively the number of Tm atoms in a given atomic column [2]. It is based on locally integrated intensities rather than peak intensities of HAADF images. The variation of the visibitily coefficient with the incident convergence angle and the image defocalisation will be studied by STEM image simulations. This integrated visibility is less sensitive to the defocus-induced blurring or to the position of the Tm atom within the thin lamella (Fig. 3).

We found that most of $\mathrm{Tm}$ atoms diffuse out of the $\mathrm{GaN}$ dots. Tm atoms are found at different positions in the AlN matrix. Above the wetting layers, Tm atoms are spread within a thickness of 14 AlN monolayers (MLs) (Fig. 1c). Above the quantum dots all the Tm are located nearly in the same plane situated at 2-3 MLs above the apex of the GaN dot, i.e. at a distance of 14 MLs from the wetting layers (Fig. 1b). An explanation is proposed based on the grow mechanism [3] of the GaN dots and AlN capping. In addition, Tm can diffuse very far from the GaN WL by following threading dislocations lines (Fig. 2).

References

[1] Y. Hori et al., Appl. Phys. Lett. 84, (2004) 206.

[2] H. Okuno et al., Appl. Phys. Lett. 96 (2010) 251908

[3] J. Coraux et al., Phys. Rev. B 74 (2006) 195302.

[4] We acknowledge the help of the French ANR Cartodop for this work. 

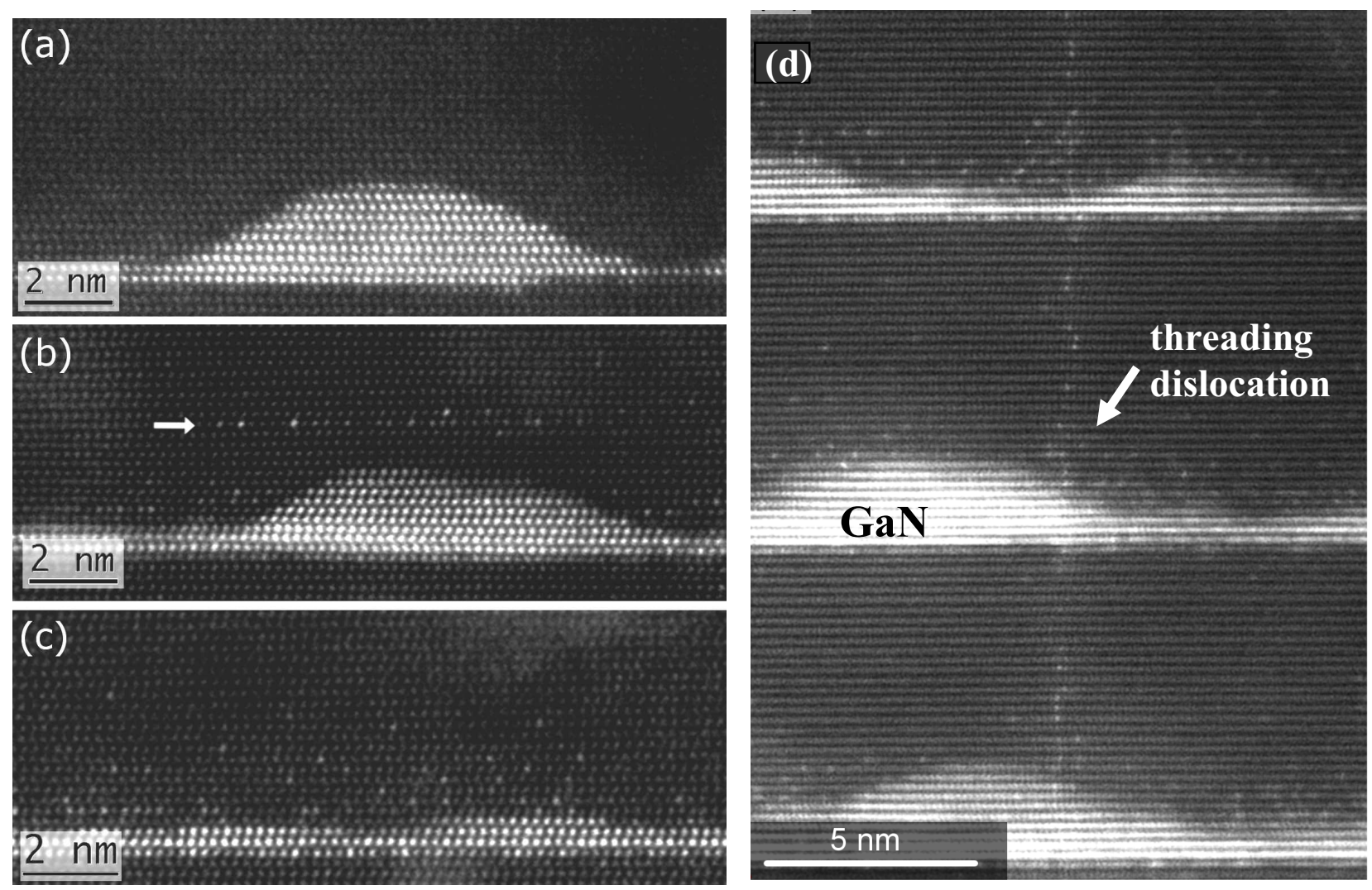

FIG. 1. High-resolution HAADF STEM images of GaN quantum dots and wetting layers embedded in AlN either taken along a $<2,-1,-1,0>$ direction ( $a-b-c)$ or along $<0,1,-1,0>$ (b).

(a) Undoped GaN dot. (b) Tm doped GaN dot. Bright spots in AlN correspond to Al columns containing one or several Tm atoms. (c) a GaN wetting layer between Tm doped GaN dots.

(d) A series of 3 layers containing $\mathrm{GaN}$ dots at the edge of a threading dislocation which is decorated by Tm atoms.

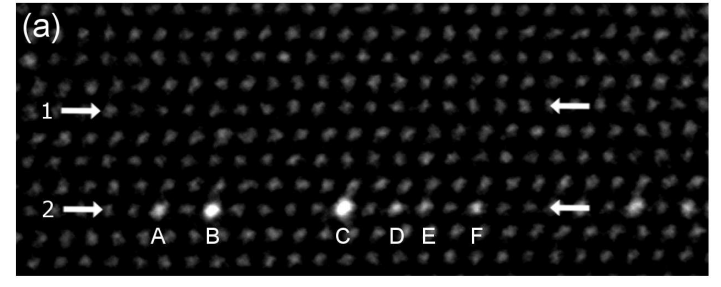

(b)
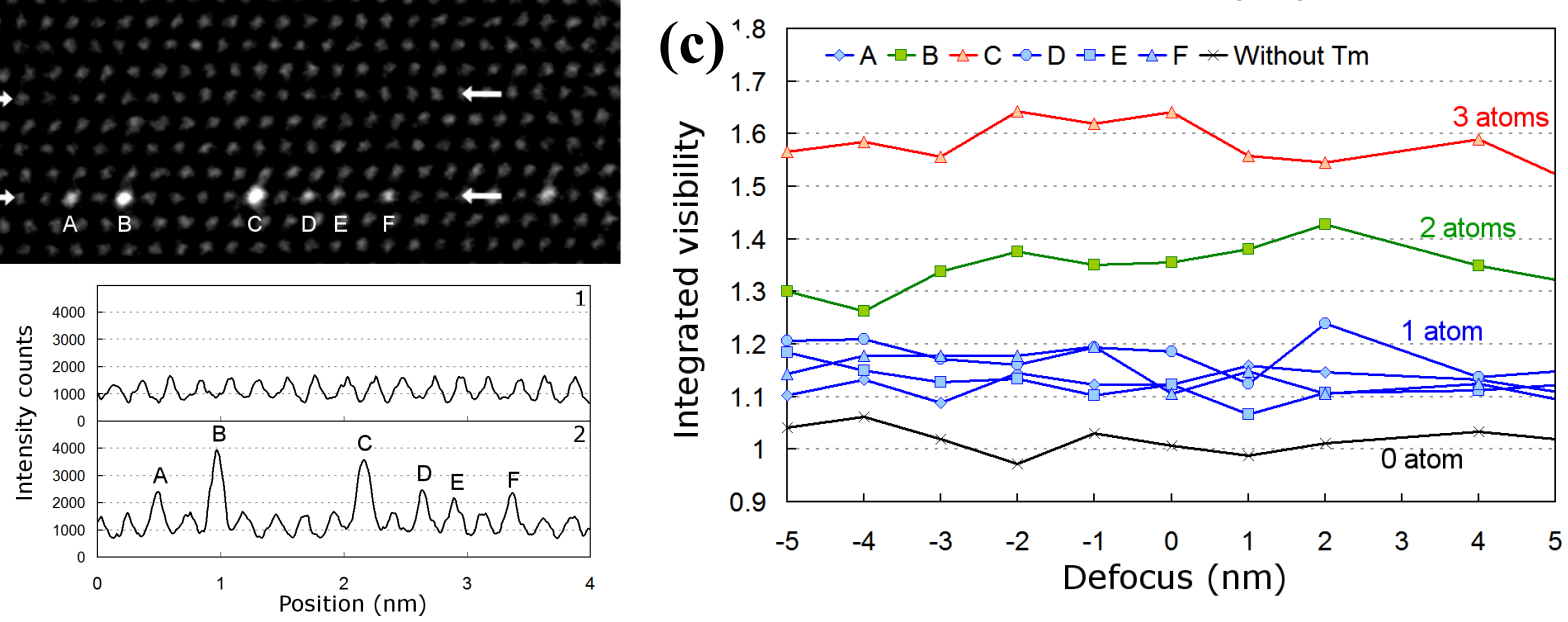

FIG. 2. (a) Zoom on the area that is pointed by an arrow in Fig. 1b. (b) Profiles of the HAADF image of Fig. 2(a) taken respectively along the lines 1 and 2. Line 2 contains Tm atoms. (c) Experimental integrated visilibity coefficients taken on atomic columns marked A-B-C-E-F, at different defocus (from a focal series). Column B contains $2 \mathrm{Tm}$ atoms, column $\mathrm{C}$ has $3 \mathrm{Tm}$ atoms. 